\title{
Effect of nasogastric tubes on nasal resistance during infancy
}

\author{
JANET STOCKS \\ Department of Paediatrics and Neonatal Medicine, Institute of Child Health, Hammersmith Hospital, London
}

SUMMARY Nasal resistance $(\mathrm{Rn})$ and total airways resistance (RAw) during nose breathing were measured in two groups of preterm infants. One group had been fed by nasogastric tube during the neonatal period, while the other had received only orogastric or bottle feeds. There were no significant differences in either Rn or RAw between the two groups, which suggests that for these infants a history of nasogastric tube feeding had no adverse effect on subsequent respiratory function. The acute effects of the nasogastric tube (NGT) were assessed by measuring Rn and RAw with and without the NGT in situ. A significant increase in both Rn and RAw occurred when the NGT was in situ, particularly in white infants, and when the NGT was passed through the larger of the two nostrils.

Nasogastric tube (NGT) feeding is commonly used for preterm infants although little is known about either the acute or long-term effect this may have on nasal resistance $(\mathbf{R n})$. Previous work has shown that approximately half the total airways resistance (RAw) in white infants is due to the resistance of the nasal passages. ${ }^{1}$ Consequently, as most neonates are obligatory nose breathers, any form of nasal obstruction could have significant effects on the overall function of the respiratory system.

This study was carried out to determine whether NGT feeding had any adverse effect on subsequent $\mathrm{Rn}$ or RAw, and to measure the effect on airflow resistance of an NGT in situ.

Department of Paediatrics, Institute of Child Health, Hammersmith Hospital

JANET STOCKS, research fellow

\section{Subjects}

Long-term effects of the NGT. Measurements of resting $R n$ were made in 16 preterm infants $(10$ white, 6 black) who had been fed via an NGT for varying periods of time (NGT group), and in 10 preterm infants (6 white, 4 black) who had been fed only orogastrically or by bottle (oral group). Details of the infants are summarised in Table 1. Concurrent measurements of thoracic gas volume (TGV) and RAw were made in 20 of these infants.

There was no history of cardiopulmonary disease in any of the infants in the oral group, whereas 4 of the NGT infants had suffered from respiratory distress syndrome during the early neonatal period. However, since none of these infants had received nasotracheal intubation and all had regained entirely normal lung function by the time of testing, they were included in the study group.

Table 1 Summary of long-term effects of nasogastric tube feeding on respiratory function. (Mean values $\pm S E M$ )

\begin{tabular}{|c|c|c|c|c|}
\hline & \multicolumn{2}{|l|}{ White babies } & \multicolumn{2}{|l|}{ Black babies } \\
\hline & $N G T(n=10)$ & Oral $(n=6)$ & $N G T(n=6)$ & Oral $(n=4)$ \\
\hline $\begin{array}{l}\text { Gestational age (weeks) } \\
\text { Postnatal age (days) } \\
\text { Body weight at time of testing (kg) } \\
\text { Duration of NGT feeding (days) } \\
\text { Nasal resistance (kPa/l per second) } \\
\text { Nasal resistance as a \% total airways resistance } \\
\text { Specific airways conductance during nose } \\
\text { breathing (kPa/s) }\end{array}$ & $\begin{array}{l}31 \cdot 5 \pm 0 \cdot 8 \\
32 \cdot 5 \pm 5 \cdot 8 \\
2 \cdot 01 \pm 0 \cdot 07 \\
27.6 \pm 5 \cdot 3 \\
1 \cdot 89 \pm 0 \cdot 10 \\
50 \cdot 7 \pm 2 \cdot 4(\mathrm{n}=8) \\
3 \cdot 8 \pm 0 \cdot 1(\mathrm{n}=8)\end{array}$ & $\begin{aligned} 32 \cdot 3 & \pm 1 \cdot 1 \\
25 \cdot 0 & \pm 7.4 \\
2 \cdot 11 & \pm 0.06 \\
- & \\
1 \cdot 88 & \pm 0 \cdot 15 \\
48 \cdot 6 & \pm 3 \cdot 7(n=4) \\
3 \cdot 5 & \pm 0 \cdot 1(n=4)\end{aligned}$ & $\begin{array}{l}32.6 \pm 0.6 \\
29.4 \pm 5.0 \\
1 \cdot 84 \pm 0.07 \\
18.9 \pm 3.6 \\
0.90 \pm 0.08 \\
28 \cdot 7 \pm 3.3 \\
4 \cdot 7 \pm 0.3\end{array}$ & $\begin{array}{r}33 \cdot 0 \pm 1 \cdot 6 \\
11 \cdot 5 \pm 4 \cdot 2 \\
2 \cdot 04 \pm 0 \cdot 18 \\
\overline{1.04} \pm 0 \cdot 12 \\
34 \cdot 0(\mathrm{n}=2) \\
5 \cdot 2(\mathrm{n}=2)\end{array}$ \\
\hline
\end{tabular}

Value of resistance expressed in $\mathrm{kPa} / 1$ per second and should be multiplied by a factor of 10 , to obtain resistance in $\mathrm{cmH}_{2} \mathrm{O} / 1$ per second, whereas specific conductance should be divided by 10 to obtain values in $\mathrm{cmH}_{2} \mathrm{O} / \mathrm{s}$. 
Acute effects of the NG'T. Attempts to measure Rn with and without a feeding catheter (FG 5) in situ were made in 21 infants, but satisfactory results were obtained in only 7 infants due to the prolonged nature of the test, and the disturbance caused to the infant by passing the NGT. Of these infants, 4 had previously only been fed orally, while the remaining 3 had received NGT feeds (Table 2).

This study was approved by the District Ethical Committee and informed parental consent was obtained before it started.

\section{Method}

All measurements were performed 30-60 minutes postprandially, and the infants were lightly sedated with chloral hydrate syrup $(50 \mathrm{mg} / \mathrm{kg})$

Long-term effects. $\mathrm{Rn}$ was measured using an adaptation of the posterior rhinomanometric method which has been described; ${ }^{1}$ changes in transnasal pressure are measured, using a small mouth balloon positioned in the posterior buccal cavity, and are related to simultaneous changes in the rate of air flow measured with a pneumotochograph attached to a face mask.

Whenever possible, concurrent measurements of TGV and RAW were made, using an adaption of the plethysmographic technique which has been described. ${ }^{2-3}$ As infants breathe naturally through their noses, and rarely resort to mouth breathing, total RAw in infants comprises both nasal resistance and the resistance of the airways below the nasopharynx, thus giving an assessment of the overall function of the respiratory system.

Acute effects. The acute effects of the NGT were assessed by measuring resting $\mathrm{Rn}$, and then attempting to repeat the measurements with a feeding catheter (FG 5) passed through each nostril in turn, with a final measurement being made after the catheter had been removed altogether. However many infants were disturbed by this procedure, especially since satisfactory and reproducible values of resting $\mathrm{Rn}$ could take more than an hour to obtain, and take even longer with the NGT in situ. Consequently reliable measurements of $\mathrm{Rn}$ with the NGT in situ were obtained in 7 infants, only 2 of whom co-operated at every stage of the tests.

It had been hoped to assess the acute effects of the NGT on total RAw in the same 7 infants but this proved to be impossible for practical reasons. However measurements of RAw with and without the NGT in situ were made in 4 infants.

\section{Results}

Long-term effects. Resting values of $\mathrm{Rn}$ were significantly higher in white infants than in their black counterparts $(\mathrm{P}<0.001)$, and it was therefore necessary to analyse their results separately. Within

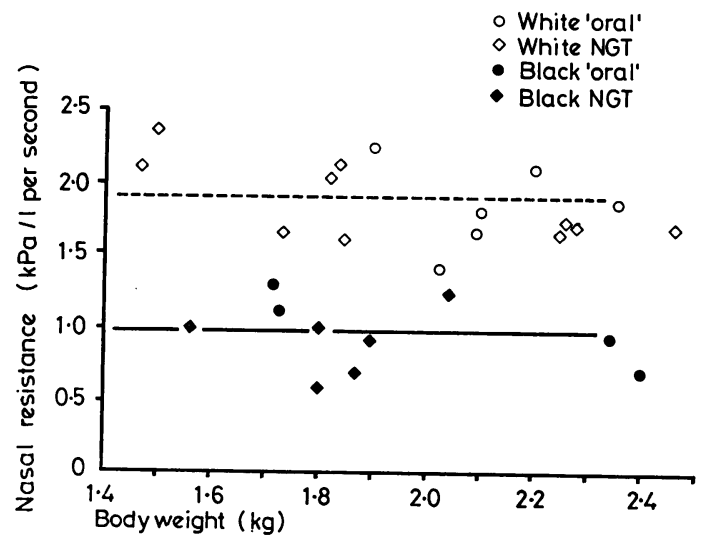

Fig. 1 Nasal resistance $(R n)$ plotted against body weight in preterm infants, showing the significantly higher values of $R n$ in white than in black infants, but the absence of any effect of previous nasogastric tube feeding on $R n$ within each racial group.

Table 2 Acute effects of the nasogastric tube on nasal resistance in preterm infants

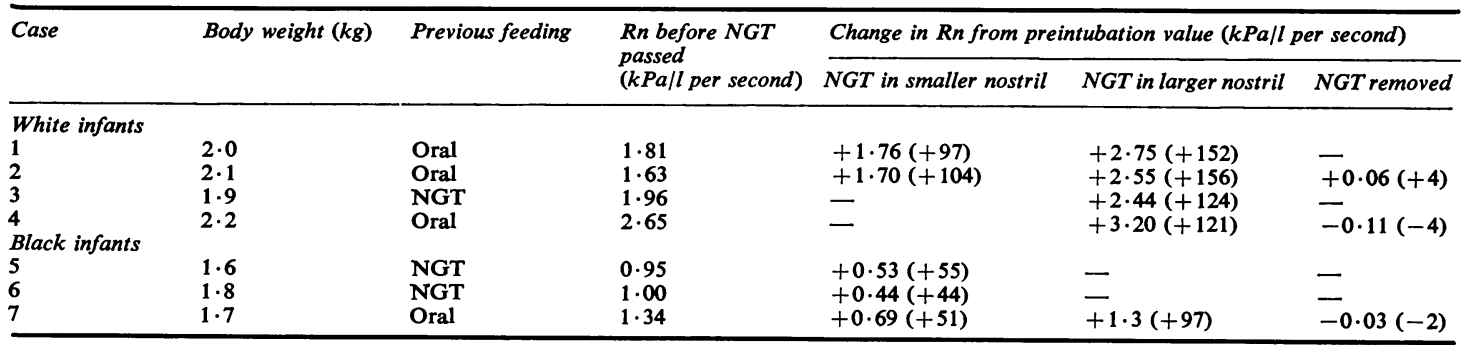

Oral, fed by orogastric tube or bottle, or both; NGT, nasogastric tube feeding.

$1 \mathrm{kPa}=10 \mathrm{cmH}_{2} \mathrm{O}$ pressure.

Percentages are given in parentheses. 
each racial group there was no significant difference between the NGT and oral groups with respect to resting Rn (Table 1, Fig. 1). There was a tendency for $R n$ to decrease with increasing body weight in the white infants $(r=-0 \cdot 5)$, but this relationship was not significant in the small range of infants studied $(P=0 \cdot 06)$, and was not apparent in the black infants.

In each infant, $\mathrm{Rn}$ was calculated as a percentage of total RAW (where RAw represents the sum of the resistances of both the nasal passages and the airways below the nasopharynx). Once again, there was a significant difference $(P<0 \cdot 001)$ between the white infants in whom $\mathrm{Rn}$ represented $50.0 \pm 6.8 \%$ (SD) of total RAw, and the black infants in whom Rn contributed a mean of only $30 \cdot 5 \pm 6 \cdot 9 \%$ (SD); whereas differences in previous feeding had no significant effects (Fig. 2, Table 1).

Finally, in order to obtain an assessment of overall airways calibre, corrected for differences in lung size, specific airways conductance (SGAw) was calculated by dividing airways conductance $(\mathrm{GAw}=$ $1 /$ RAw) by the infant's TGV. Within each racial group, SGAw was found to be unaffected by previous NGT feeding (Table 1).

Acute effects. In all the infants in whom it was possible to measure $\mathrm{Rn}$ with the NGT in situ, it was found to be easier to pass the NGT through one of the nostrils (designated the larger); this was the right nostril in 5 infants and the left in the remaining 2.

When the NGT was passed through the smaller nostril, mean $\mathrm{Rn}$ increased by $101 \%$ in white infants and by $50 \%$ in black ones, whereas when it was placed in the larger nostril, the increase in $\mathbf{R n}$ was considerably greater, being $138 \%$ in the white

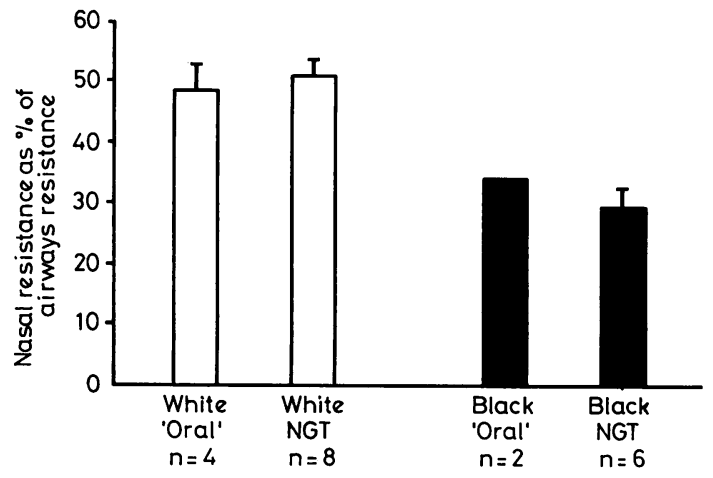

Fig. 2 Nasal resistance as a percentage of total airways resistance during nose breathing in each of the study groups. The mean $\pm S E M$ is shown for each group, except the black oral group as airways resistance was only measured in 2 infants. infants and $97 \%$ in a black infant (Table 2, Fig. 3). Resting levels of $\mathrm{Rn}$ were considerably lower in the black infants and the presence of the NGT caused a much smaller rise in $\mathrm{Rn}$ (both in absolute terms and percentage increase). In the 3 infants in whom it was possible to repeat the measurements after the NGT had been removed (Cases 2, 4, and 7, Table 2), Rn was found to be very similar to the original basal value.

The acute effects of the NGT on total airways resistance in 4 white preterm infants are shown in Fig. 4. Mean Raw rose by $49.5 \%$ when the NGT was in the larger nostril, and by $32.5 \%$ when it was in the smaller one.

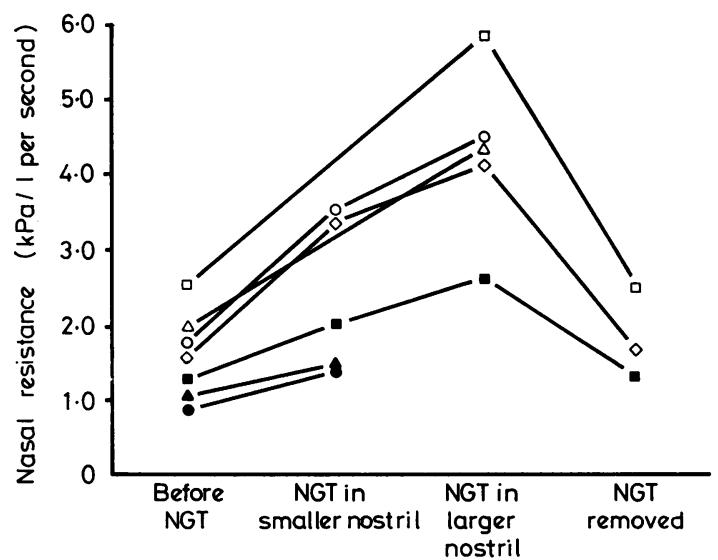

Fig. 3 Acute effects of the nasogastric tube on nasal resistance. Individual infants are represented by different symbols; open symbols representing white infants, and closed symbols black ones.

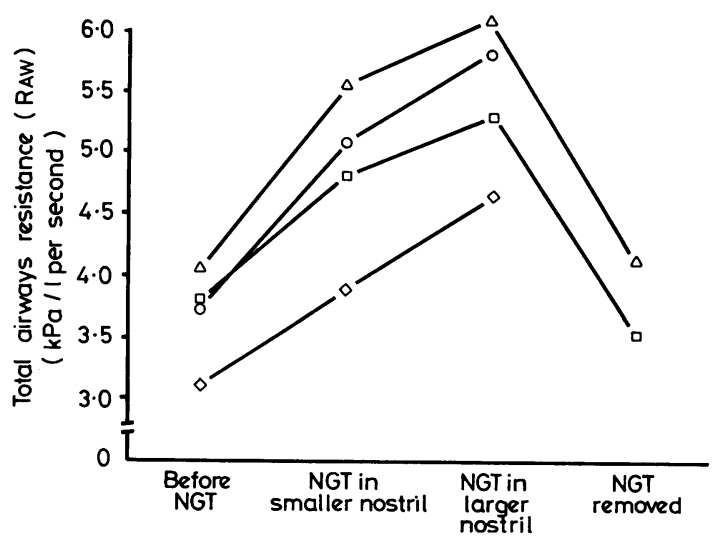

Fig. 4 Acute effects of the nasogastric tube on total airways resistance in white preterm infants. Individual infants are represented by different symbols. 


\section{Discussion}

Long-term effects. Anatomical differences in nasal structure probably explain the higher $\mathrm{Rn}$ found in white than in black infants, which was also found in a previous study. ${ }^{1}$ Differences in $\mathrm{Rn}$ between black and white infants have been shown to be responsible for the lower RAw and higher SGAw during nose breathing in black infants (Table 1), with no evidence of any racial differences in the calibre of the lower airways. ${ }^{1}$

Rn was found to represent approximately $50 \%$ of total RAw in the white preterm infants, irrespective of feeding history, which is very similar both to the values reported for infants during the first year of life, ${ }^{1}$ and to those given for adults, ${ }^{4-5}$ suggesting that this relationship remains relatively constant throughout life.

Thus comparison of resting results between the NGT and oral groups of babies showed no great differences with respect to any of the parameters measured, suggesting that for these infants, previous NGT feeding did not appear to have had an adverse effect on subsequent Rn or RAW.

Acute effects. During the course of this study it was noted that one nostril always appeared to be larger than the other, and that the greatest increase in Rn occurred when the NGT was passed through the larger nostril. This was presumably due to the fact that when the larger nostril was obstructed by the NGT, the infant was forced to breathe through the smaller nostril whose resistance would be considerably greater. Since this effect was noted whether the infant had been fed orally or nasogastrically, the discrepancy in nostril size cannot be attributed to possible effects of previous NGT feeding. A pronounced difference in the calibre of the 2 nasal passages has been reported both in infants ${ }^{6}$ and adults. ${ }^{7}$ In addition, Lacourt and Polgar ${ }^{6}$ noted cyclic variations in the calibre of the 2 nostrils.

If the overall calibre of the nostrils was greater in black infants, as the resting values of $R n$ suggest, the presence of the NGT would cause relatively less obstruction and hence a smaller increase in $\mathrm{Rn}$ than in white infants, as indeed was found.

In all the infants in whom it was possible to measure Rn or RAW after the NGT hadbeen removed, very similar values to the original basal measurements were found. However, on one occasion the presence of the NGT caused a considerable degree of nasal secretion, which persisted after removal of the tube. Unfortunately it was not possible to measure nasal resistance in this infant as he became distressed and snuffly, but this observation does suggest that on occasions, the NGT may cause not only a degree of physical obstruction but irritation to the nasal mucosa resulting in increased secretions.

The experiments performed to assess the effects of the NGT on total RAw showed that there was a significant increase in RAW when the NGT was in situ, this effect being more pronounced when the NGT was in the larger nostril (Table 3, Fig. 4). However, whereas mean $\mathrm{Rn}$ increased by $1.7 \mathrm{kPa} / 1$ per second $\left(17 \mathrm{cmH}_{2} \mathrm{O} / 1\right.$ per second) when the NGT was in the smaller nostril, and by $2.7 \mathrm{kPa} / 1$ per second ( $27 \mathrm{cmH}_{2} \mathrm{O} / 1$ per second) when the tube was in the larger nostril, the corresponding mean changes in RAW were only 1.3 and $1.9 \mathrm{kPa} / 1$ per second (13 and $19 \mathrm{cmH}_{2} \mathrm{O} / 1$ per second) respectively. Whether this discrepancy can be attributed to the fact that the effects of the NGT on Rn and RAw were assessed in different groups of babies and that the numbers were somewhat limited, or to a degree of experimental error is not known. It is unlikely that the relatively smaller increase in RAw was due to the infant partly changing to mouth breathing in an attempt to overcome the obstruction when the NGT was in situ as most preterm infants are unable to mouth breathe even if both nostrils are completely obstructed, unless they cry. All these measurements were taken during quiet breathing. In addition had the infants succeeded in mouth breathing when the NGT was in situ, one would have expected to see a fall in RAW below the resting values, instead of the significant increase that was measured.

It is possible that some form of compensatory reflex action in the airways below the nasopharynx could have occurred in response to the partial nasal obstruction. Rattenborg ${ }^{8}$ suggested that total RAW could be kept relatively constant in the presence of various external resistances by altering the position of the larynx, but this function of the larynx could not be confirmed by Spann and Hyatt ${ }^{9}$ and is at variance with results obtained from this laboratory where it was shown that if variable resistances were added to the rebreathing apparatus, the measured RAW (apparatus plus infant) rose by almost identical increments. ${ }^{2}$

Lacourt and Polgar ${ }^{6}$ found that whereas resting values of $\mathbf{R p}$ (pulmonary resistance) remained relatively constant throughout the day, $R n$ varied considerably. Consequently, they concluded that the resistance below the nasopharynx must have been changing inversely and simultaneously with the changes in Rn, presumably due to some compensatory reflex phenomenon. However, the technique that they used for measuring $\mathrm{Rn}$ involved occluding alternate nostrils and depended on the patency of one nostril being totally unaffected (either mechanically or reflexly) by the occlusion of the other, an assumption which is unlikely to be valid 
according to the work of Brown, ${ }^{7}$ and which may explain the pronounced variability of $\mathrm{Rn}$ in the presence of consistent values of $R p$, an observation which has not been confirmed in this laboratory.

Widdicombe ${ }^{10}$ showed that stimulation of epipharyngeal receptors by a jet of air and other stimuli could lead to significant bronchodilatation, although others have found that in some instances different types of mechanical stimulation of the nasopharynx can induce bronchoconstriction. ${ }^{11}$ Obviously further work is needed to elucidate the effects of such reflexes in infants.

However, if such a compensatory reflex exists in infants, the results of the present study suggest that it is not strong enough to negate the effects of nasal obstruction, and that the presence of an NGT will cause a significant increase in total RAw, at least in white preterm infants.

In the past some studies of pulmonary resistance $(\mathrm{Rp}=$ airways plus tissue resistance), using the oesophageal balloon technique, have been performed with the catheter passed through the nostril. ${ }^{12-14}$ However, since the presence of any tube is likely to cause an increase in $\mathrm{Rp}$ - the extent of which will vary according to the size and race of the infant, the dimensions of the catheter, and the choice of nostrilsuch measurements of lung mechanics should obviously be performed with the catheter passed orally.

In conclusion, while nasogastric feeding may often be the most convenient and suitable method of feeding small preterm infants and does not appear to have any adverse effect on their subsequent respiratory function, a considerable degree of nasal obstruction can occur while the tube is in situ. Efforts should therefore be made to use the smallest feeding tube possible, to pass it through the smaller of the two nostrils (providing this does not necessitate undue force), to ensure that the opposite nostril remains completely unobstructed and, wherever possible, to avoid NGT feeding in infants who are already suffering from respiratory distress.

\section{References}

1 Stocks J, Godfrey S. Nasal resistance during infancy. Respir Physiol 1978; 34: 233-46.

2 Stocks J, Levy N M, Godfrey S. A new apparatus for the accurate measurement of airway resistance in infancy. J Appl Physiol 1977; 43: 155-9.

3 Stocks J, Godfrey S. Specific airway conductance in relation to post conceptional age during infancy. $J$ Appl Physiol 1977; 43: 144-54.

4 Unno T, Nelson J R, Ogura J H. The effect of nasal obstruction on pulmonary airway and tissue resistance. Laryngoscope 1968; 78: 1119-39.

5 Butler J. The work of breathing through the nose. Clin Sci Mol Med 1960; 19: 55-62.

6 Lacourt G, Polgar G. Interaction between nasal and pulmonary resistance in newborn infants. J Appl Physiol 30: 870-3.

7 Brown E A. The measurement of the resistance of the nasal passages to the movement of air. II. Increased resistance, mechanical and reflex, subsequent to the occlusion of alternate nostrils. Rev Allergy 1967; 21 : 852-7.

8 Rattenborg C. Laryngeal regulation of respiration. Acta Anaesthesiol Scand 1961 ; 5: 129-40.

${ }^{9}$ Spann R W, Hyatt R E. Factors affecting upper airway resistance in conscious man. J Appl Physiol 1971; 31: 708-12.

10 Widdicombe J G. Defensive mechanisms of the respiratory system. In: Widdicombe J G, ed. Respiratory physiology, part 2. Baltimore: University Park Press, 1977; 291-315.

11 Kaufman J, Chen J C, Wright G W. The effect of trigeminal resection on reflex bronchoconstriction after nasal and nasopharyngeal irritation in man. Am Rev Respir Dis $1970 ; 101$ : 768-9.

12 Swyer P R, Reiman R C, Wright J J. Ventilation and ventilatory mechanics in the newborn. $J$ Pediatr 1960; 56: 612-22.

13 Ahlström H, Jonson B. Pulmonary mechanics in infants. Methodological aspects. Scand J Respir Dis 1974; 55: $129-40$.

14 Hjalmarson O, Olsson T, Riha M. Mechanics of breathing in newborn infants with pulmonary disease. II. Theoretical considerations and methods. Acta Paediatr Scand (Suppl) 1974; Supplement 247, 6-25.

Correspondence to Dr Janet Stocks, Department of Paediatrics and Neonatal Medicine, Hammersmith Hospital, Du Cane Road, London W12 0HS.

Received 8 March 1979 